\title{
Offline handwritten signature verification using Alpha Cut of Triangular Fuzzy Number (TFN)
}

\author{
Rozaimi Zakaria ${ }^{1 *}$, Abd. Fatah Wahab ${ }^{1}$ and Jamaludin Md. Ali ${ }^{2}$ \\ ${ }^{1}$ Department of Mathematics, Faculty of Science and Technology, Universiti Malaysia Terengganu, 21030 Kuala Terengganu, Terengganu, Malaysia. \\ ${ }^{2}$ School of Mathematical Science, Universiti Sains Malaysia, 11800 USM, Pulau Pinang. \\ Received 29 June 2010, Revised 15 November 2010, Accepted 20 November 2010, Available online 25 November 2010
}

\begin{abstract}
A new approach in verification of offline handwriting signature had been proposed. This approach use alpha-cut of triangular fuzzy number in verification of the signature where the value of alpha-cut will reduce the interval of confidence fuzzy interval. The best value of alpha-cut will make the confidence fuzzy interval in the smallest interval which the best confidence fuzzy interval consists less number of signature from the present number of the signature. Then, defuzzification method is used to achieve defuzzification signature. The signatures modeled by piecewise fuzzy interpolation rational cubic Bezier curve.
\end{abstract}

| Alpha-cut | Triangular fuzzy number | Confidence fuzzy interval | Fuzzy interpolation rational cubic Bezier curve |

(B) 2010 Ibnu Sina Institute. All rights reserved. http://dx.doi.org/10.11113/mjfas.v6n2.199

\section{INTRODUCTION}

Handwritten signature is most widely accepted in personal attributes for identity verification. It is also a symbol of authorization, especially in the prevalence of credit cards and bank cheques that had been long become the target of falsification. Therefore, with the exists and growing demand for processing of individual identification faster and more accurately, the design of an automatic signature verification system faces a real challenge.

Handwritten signature verification can be divided into two parts that are online and offline signature verification. Online verification refers to a process that signature owner use a special digital pen called stylus to create his or her signature. While, for offline verification is just deals with signature image acquired by a scanner or a digital camera.

Generally, offline signature verification is a challenging problem. Contrary as the online signature which captured directly as the handwriting trajectories in digital form that can be analyzed very fast. For offline signature, the signatures captured manually using a few step of digital documentation before the analysis can be done.

During the last few years, researchers have made great effort on offline signature verification. Huang and Yan proposed a method for verification of offline signature is based on geometric and neural network classification [2]. Kalera and friends describes the signature verification and

Corresponding author at: Department of Mathematics, Faculty of Science and Technology, Universiti Malaysia Terengganu, 21030 Kuala Terenggganu, Terengganu, Malaysia.

E-mail address: rozaimi_z@yahoo.com (Rozaimi Zakaria) identification in offline environment based on a quasimultiresolution technique using GSC (Gradient, Structural and Concavity) features for feature extraction [3]. Then, Shanker and Rajagopalan proposed a signature verification system based on Dynamic Time Warping (DTW) which works by extracting the vertical projection feature from signature images and by comparing reference and probe feature template using elastic matching [6].

In this paper, a new approach is proposed in offline signature verification on alpha-cut of triangular fuzzy number (TFN) [4, 9, 10 and 11]. The original (crisp) signature become main subject to create TFN of signature which left and right TFN are left (lower bound) fuzzy signature and right (upper bound) fuzzy signature that both constructed through fuzzy interpolation rational cubic Bezier curve model. Then, the operation alpha-cut against fuzzy signature after defined by TFN will reduce the interval between left(lower bound) fuzzy signature and right (upper bound) fuzzy signature. The reducing interval known as confidence fuzzy interval which means if the value of alpha is increase as the confidence fuzzy is decrease. The suitable confident fuzzy interval consists less number of the signature (at least one signature) from total present number of signatures. There are given several of offline signatures which same as the original with slight differences. Therefore, the signatures analyzed through this approach.

The remainder of this paper is organized as follows: Section 2 is the preliminaries; Section 3 is discusses the fuzzy signature modeling with crisp(original) signature and left and right fuzzy signature; Section 4 is the alpha cut implementation in fuzzy signature modeling; Section 5 is 
the analysis and result; Section 6 is the defuzzification method; and Section 7 is the conclusion.

\section{PRELIMINARIES}

The fuzzy set theory was introduced firstly by Zadeh in year 1965 [8] which present the basics idea of fuzzy set theory. The other implementation of fuzzy set theory is fuzzy number concept which is used in order to solve the problem in define the uncertainty data.

\section{Definition 1: [7] (Fuzzy Set)}

Let $X$ be a universal set and $A \subset X$. Set $A$ called fuzzy set denoted by $\vec{A}$ if for every $x \in X$ there exists $\mu_{A}: X \rightarrow[0,1]$ a form of membership function that characterizing the membership grade for every element of $A$ in $X$ defined by

$$
\mu_{A}(x)=\left\{\begin{array}{cl}
1 & \text { if } x \in A \text { (full membership) } \\
c \in(0,1) & \text { if } x \overrightarrow{\in A} \text { (non-full membership) } \\
0 & \text { if } x \notin A \text { (non-membership) }
\end{array}\right.
$$

So, fuzzy set $\vec{A}$ can be written as $\vec{A}=\left\{\left(x, \mu_{A}(x)\right)\right\}$ which is $\vec{A}$ in $X$ is a set of order pair denoted generically by $x$ in $X$ with grade of membership $\mu_{A}(x)$ in $[0,1] . \square$

\section{Definition 2: [4, 9, 10 and 11] (Fuzzy Number)}

A fuzzy set $\vec{A}$ is called a fuzzy number if the following conditions are satisfied

(i) There exist $x \in R$, such that $\mu_{A}(x)=1$

(ii) For any $\alpha \in(0,1]$ the set $\left\{x: \mu_{A}(x) \geq \alpha\right\}$ is a closed interval denoted by $\left\langle\bar{A}_{\alpha}^{-}, A, \vec{A}_{\alpha}^{+}\right\rangle$.

Then, fuzzy number is actually fuzzy set defined on the set of real numbers which characterized by mean of membership function. It represents information "about" or "around" such number which has unique modal crisp value, convex, left-right continuous, increasing and decreasing.
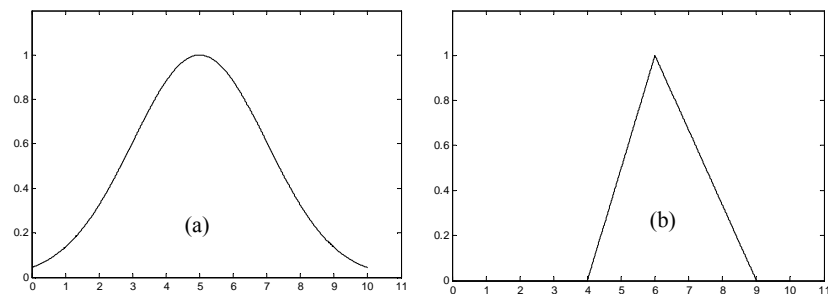

Figure 1: (a). Gaussian Fuzzy Number about " 5 " and (b) Triangular Fuzzy Number about "6".

\section{Definition 3: [7] (Fuzzy Control Point)}

Fuzzy set $\vec{P}$ in a space of $S$ said set of fuzzy control point if for every $\alpha$-level set was chosen, there exists pointed interval that is $P=<P_{i}^{\leftarrow}, P_{i}, P_{i}^{\rightarrow}>$ in $S$ with every $P_{i}$ is crisp point and membership function $\mu_{P}: S \rightarrow[0,1]$ which defined as $\mu_{P}\left(P_{i}\right)=1$,

$$
\begin{aligned}
& \mu_{P}\left(P_{i}^{\leftarrow}\right)= \begin{cases}0 & \text { if } P_{i}^{\leftarrow} \notin S \\
c \in(0,1) & \text { if } P_{i}^{\leftarrow} \vec{\in} S \text { and } \\
1 & \text { if } P_{i}^{\leftarrow} \in S\end{cases} \\
& \mu_{P}\left(P_{i}^{\rightarrow}\right)= \begin{cases}0 & \text { if } P_{i}^{\rightarrow} \notin S \\
k \in(0,1) & \text { if } P_{i}^{\rightarrow} \ddot{\in} S \\
1 & \text { if } P_{i}^{\rightarrow} \in S\end{cases}
\end{aligned}
$$

with $\mu_{P}\left(P_{i}^{\leftarrow}\right)$ and $\mu_{P}\left(P_{i}^{\rightarrow}\right)$ are left membership grade value and right membership grade value respectively and generally written as

$$
\vec{P}=\left\{\vec{P}_{i}: i=0,1,2, \ldots, n\right\}
$$

for every $i, \vec{P}_{i}=<\vec{P}_{i}^{\leftarrow}, P_{i}, \vec{P}_{i}^{\rightarrow}>$ with $\vec{P}_{i}^{\leftarrow}, P_{i}$ and $\vec{P}_{i}^{\rightarrow}$ are left fuzzy control point, crisp control point and right fuzzy control point respectively. This also same in define fuzzy data.

\section{Definition 4: [7] (Defuzzification)}

Let $\vec{P}$ a set fuzzy control point that defined through Definition 3. Set $\bar{P}$ named set defuzzification control point of $\vec{P}$ if for every $\vec{P}_{i} \in \vec{P}$,

$$
\bar{P}=\left\{\bar{P}_{i}\right\} \text { for } i=0,1, \ldots, n \text {. }
$$

with every $\bar{P}_{i}=\frac{<\vec{P}_{i}^{\leftarrow}, P_{i}, \vec{P}_{i}^{\rightarrow}>}{3}$.

Definition 5: [5 and 1] (Fuzzy Interpolation Rational Cubic Bezier Curve)

Let $\vec{P}_{i}, \vec{D}_{i} \in R, i=0,1, \ldots, n$, be a given set of fuzzy data points and fuzzy derivative value at $t$. Then, the fuzzy interpolation rational cubic Bezier curves if $\alpha=\beta=3$ is defined as

$$
\vec{R}(t)=\frac{\vec{F}_{0}(t) \vec{P}_{i}+\vec{F}_{1}(t) \vec{v}_{i} \vec{Q}_{i}+F_{2}(t) \vec{w}_{i} S_{i}+\vec{F}_{3}(t) \vec{P}_{i+1}}{\vec{F}_{0}(t)+\vec{F}_{1}(t) \vec{v}_{i}+\vec{F}_{2}(t) \vec{w}_{i}+\vec{F}_{3}(t)}
$$

with the fuzzy blending functions given by 


$$
\begin{aligned}
& \vec{F}_{0}(t)=(1-t)^{2}(1+(2-\alpha) t) \\
& \vec{F}_{1}(t)=\alpha(1-t)^{2} t \\
& \vec{F}_{2}(t)=\beta t^{2}(1-t) \\
& \vec{F}_{3}(t)=t^{2}(1+(2+\beta)(1-t))
\end{aligned}
$$

which $\vec{v}_{i}$ and $\vec{w}_{i}$ are fuzzy weights value and

$$
\begin{aligned}
& \vec{Q}_{i}=\frac{\vec{D}_{i}}{3 \vec{v}_{i}}+\vec{P}_{i} \\
& \vec{S}_{i}=\vec{P}_{i+1}-\frac{\vec{D}_{i+1}}{3 \vec{w}_{i}}
\end{aligned}
$$

with $\vec{D}_{i}$ and $\vec{D}_{i+1}$ are tangent vectors at $\vec{P}_{i}$ and $\vec{P}_{i+1}$ respectively.

This tangent vectors are defined as follow. For open curves,

$$
\begin{aligned}
& \vec{D}_{0}=2\left(\vec{P}_{1}-\vec{P}_{0}\right)-\frac{\vec{P}_{2}-\vec{P}_{0}}{2}, \\
& \vec{D}_{i}=\vec{a}_{i}\left(\vec{P}_{i}-\vec{P}_{i-1}\right)+\left(1-\vec{a}_{i}\right)\left(\vec{P}_{i+1}-\vec{P}_{i}\right), \\
& \vec{D}_{n}=2\left(\vec{P}_{n}-\vec{P}_{n-1}\right)-\frac{\vec{P}_{n}-\vec{P}_{n-2}}{2},
\end{aligned}
$$

where $\vec{a}_{i}=\frac{\left\|\vec{P}_{i+1}-\vec{P}_{i}\right\|}{\left\|\vec{P}_{i+1}-\vec{P}_{i}\right\|+\left\|\vec{P}_{i}-\vec{P}_{i-1}\right\|}, \quad i=0,1, \ldots, n$.

Thus, from Bernstein Bezier theory, the fuzzy curve lies in the convex hull of fuzzy control points $\left\{\vec{P}_{i}, \vec{Q}_{i}, \vec{S}_{i}, \vec{P}_{i+1}\right\}$ and it is variation diminishing.

\section{FUZZY OFFLINE HANDWRITTEN SIGNATURE MODELING}

In this section, offline signature digitized through scanner and then analyzed in getting point data. Fuzzy interpolation rational cubic Bezier curve was applied to model the data after the segmentation of the signature.

Given ten signatures which are have a same form with slight differences. So, the first signature is considered as a original (crisp) signature. Then the crisp signature is modeled by using fuzzy interpolation rational cubic Bezier curve. This crisp signature becomes reference to the other signatures in order to achieve the goal of this paper. The construction of this crisp signature can be referred to the method which is at Definition 5.

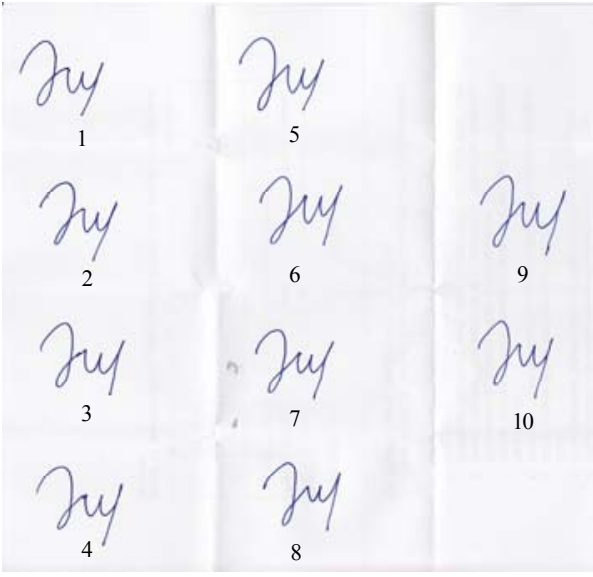

Figure 2: Ten samples of offline handwriting signature.

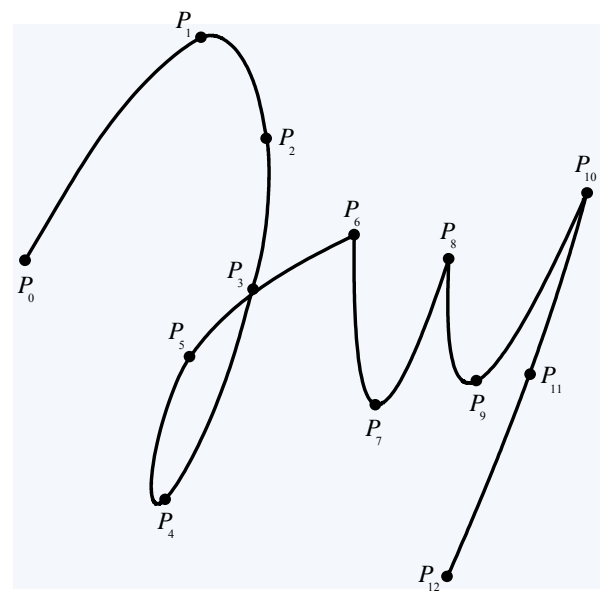

Figure 3: Crisp model of offline handwritten signature.

\section{ALPHA CUT OF TFN}

In representation of alpha-cut operation against TFN, confidence fuzzy interval is obtained based on the value of alpha as mentioned by Definition 2. When the value of alpha is increase, as the confidence fuzzy interval is decrease. Then, Figure 4 below shows the illustration.

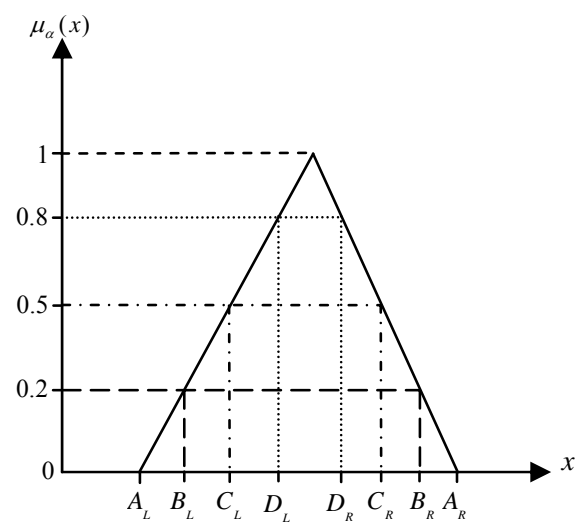

Figure 4: Alpha cut of TFN with different value of alpha.

From Figure 3, the confidence fuzzy interval defined by different value of alpha cut. Therefore, for the values of 
alpha, let say $\alpha(0.2), \alpha(0.5)$, and $\alpha(0.8)$, then their confidence fuzzy interval are $\left[B_{L}, B_{R}\right],\left[C_{L}, C_{R}\right]$, and $\left[D_{L}, D_{R}\right]$ respectively. This relationship denoted by $\left(\alpha(0.2),\left[B_{L}, B_{R}\right]\right),\left(\alpha(0.5),\left[C_{L}, C_{R}\right]\right)$, and $\left(\alpha(0.8),\left[D_{L}, D_{R}\right]\right)$ with $\left[B_{L}, B_{R}\right] \geq\left[C_{L}, C_{R}\right] \geq\left[D_{L}, D_{R}\right]$.

\section{ANALYSIS AND RESULT}

The construction of left (lower bound) and right (upper bound) of fuzzy signature is constructed before the operation of alpha cut applied which is if the different values of alpha applied, then the different confidences fuzzy interval is obtained. This relation between operation alpha cut and confidence fuzzy interval was used to specify the minimum number of signature where is in the best confidence fuzzy interval. The construction of LR-fuzzy signature (Left and Right fuzzy signature) is base on the concept of fuzzy number and also as same as LR-fuzzy control point [7] but the LR-fuzzy control point is construct based on certain points whereas the LR-fuzzy signature is constructing the curve of signature that interpolate all the data of the signature. The LR-fuzzy signature also is create based on crisp signature that modeled through Figure 3 based on Definition 5.

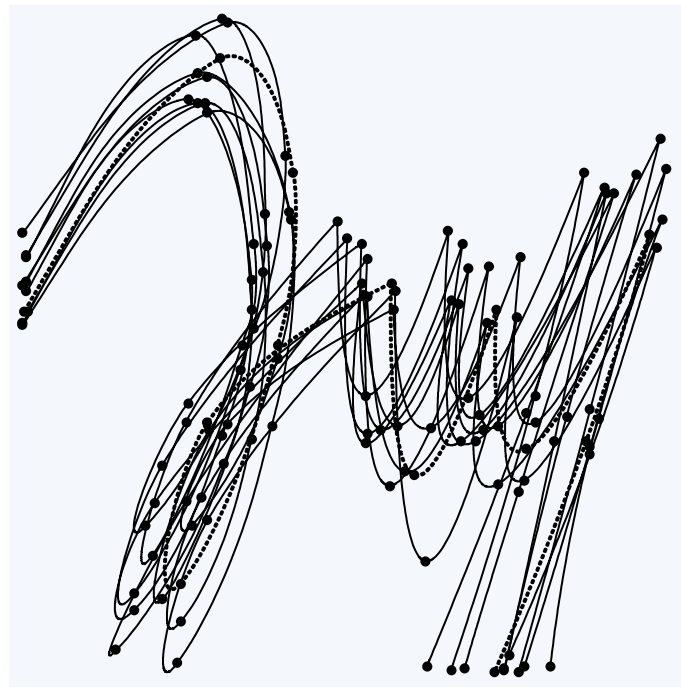

Figure 5: Ten samples signature modeled in same axis.

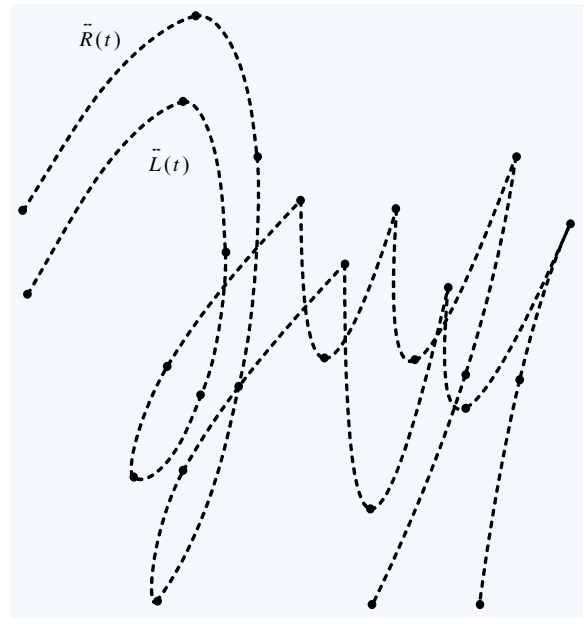

Figure 6: Left (lower bound) and right (upper bound) of fuzzy signatures taken by using alpha cut operation of TFN.

Figure 6 shows that the left (lower bound) and right (upper bound) of fuzzy signatures taken by using alpha cut operation of TFN which is the value of alpha fixed by user. In this paper, the value of alpha fixed with ( $\left.\alpha=y_{i} / 8, i=0,1,2, \ldots, 12\right)$ for every point data that lying inat the signature. The alpha value is fixed as defined before because the alpha value gives the confidence fuzzy interval where at least one signature lying in the confidence fuzzy interval which is constructed by the fixed value of alpha.

From Figure 6, the confidence of fuzzy interval between $\vec{L}(t)$ and $\vec{R}(t)$ is created in order to reduce the number of signatures and at the same time this confidence fuzzy interval will specify the best signature that is lying in this whole interval. Then, if any signature that is lying in this confidence fuzzy interval, then the signature is accepted and will analyzed, but if the signature does not lying in the confidence fuzzy interval included any segmentation of it, then the signature is rejected.

Based on Figure 5, all the ten samples of offline handwritten signatures are marked by number 1 until 10 . For each of nine samples signatures are modeled and tested in confidence fuzzy interval with fixed alpha value that is determined by alpha cut operation of TFN excluding the first signature which is the crisp signature. After every of the nine signatures are tested, there is only 8th signature is accepted, while the rest are rejected. 


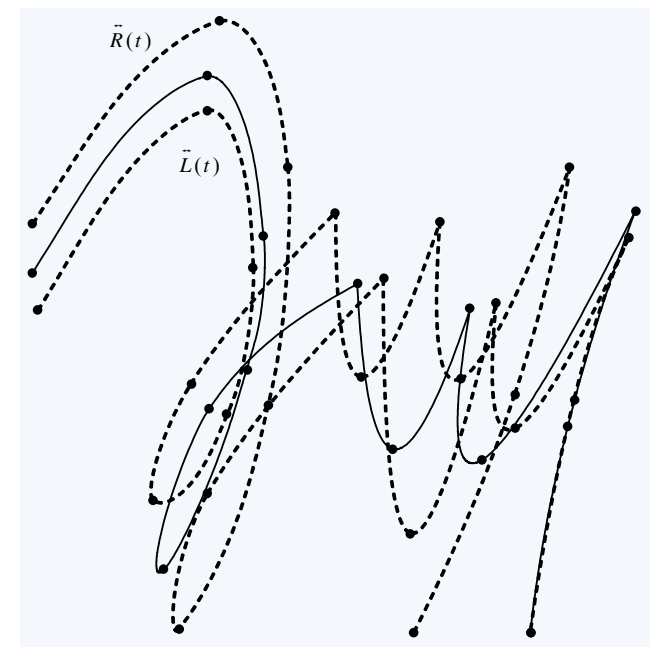

Figure 7: The 8th signature that is lying in confidence fuzzy interval of fuzzy offline handwritten signature which is accepted.

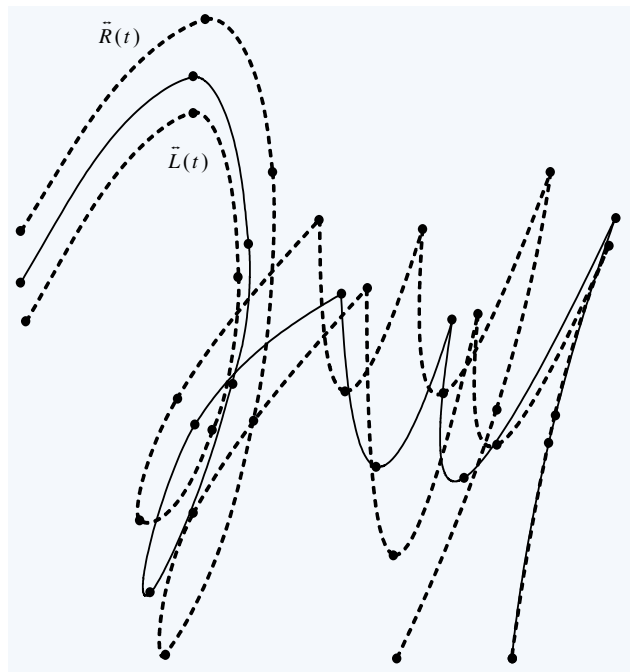

Figure 8: The 5th signature that is not in confidence fuzzy interval of fuzzy offline handwritten signature.

\section{DEFUZZIFICATION METHOD}

After the signatures were analyzed, the left (lower bound), right (upper bound) and 8th signature as in Figure 7 defuzzified to get the defuzzify offline handwritten signature. By using the Definition 4, the defuzzify offline handwritten signature was obtained which is illustrated by Figure 9.

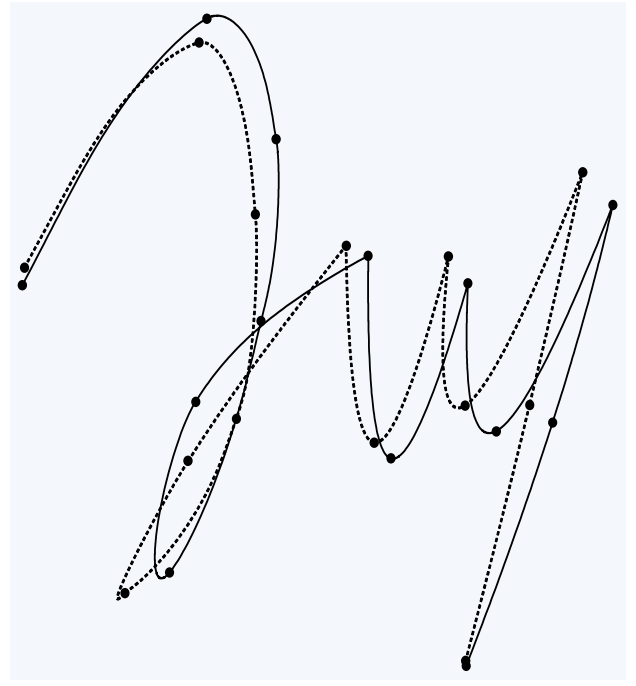

Figure 9: Defuzzify offline handwritten signature (dash) and crisp (original) offline handwritten signature.

From Figure 9, both defuzzify and crisp (original) offline handwritten signature plotted in the same axis. The using defuzzification method is to get a single signature after the analyzing as in Figure 7.

\section{CONCLUSION}

A new approach of verification offline signature using alpha cut of TFN has been constructed. Fuzzy interpolation rational cubic Bezier curve model was applied in order to design the offline handwritten signature. Then, the operation of alpha-cut was applied to construct the confidence fuzzy interval based on the alpha value. The verification of signatures was done by the fixed interval between left and right fuzzy signature to create confidence fuzzy interval that is the signature must exactly within along the fixed interval for the signature to be accepted. Therefore, if the confidence fuzzy interval increase, then more signature can be accepted and otherwise.

\section{ACKNOWLEDGEMENT}

We would like to thank the Ministry of Higher Education (MOHE) for the FRGS Vote No. 59135. The first author would also like to acknowledge Research Management Innovation Centre, Universiti Malaysia TERENGGANU (UMT) for research facilities. 


\section{REFERENCES}

[1] Azhar Ahmad dan Jamaludin Md. Ali, Mencirikan Lengkung Kubik Nisbah Alternatif. MATEMATIKA, 22(1) (2006) 77-89.

[2] K. Huang and H. Yan, Off-line signature verification based on geometric feature extraction and neural network classification. Pattern Recognition, 30(1) (1997) 9-17.

[3] M.K. Kalera, S. Srihari and A. Xu, Offline Signature Verification and Identification Using Distance Statistic. International Journal of Pattern Recognition and Artificial. 18(7) (2004) 1339-1360.

[4] G.J. Klir and B. Yuan, Fuzzy Sets and Fuzzy Logic: Theory and Applications. Prentice-Hall, Englewood Cliffs, NJ, 1995.

[5] M. Sarfraz, Z. Habib, and M. Hussain, Piecewise interpolation for designing of parametric curves. Information Visualization. Proceedings. IEEE Conference on 29-31 July 1998, (1998) $307-313$.

[6] A.P. Shanker and A.N. Rajagopalan, Offline signature verification using DTW. Pattern Recognition Letters, 28(12) (2007) 1407-1414.

[7] A.F. Wahab, J.M. Ali, A.A. Majid, and A.O.M. Tap,. Fuzzy set in geometry in modeling. Proceeding of International Conference on Computer Graphics, Imaging, and Visualization, 2004. CGIV 2004, 26-29 July 2004. IEEE Computer Society. (2004) 227-232.

[8] L.A. Zadeh, Fuzzy sets. Information and Control. 8(3) (1965) 338-353.

[9] H.J. Zimmermann, Fuzzy Set Theory and its Applications. Kluwer Academic, Boston, M.A, 1985.

[10] D. Dubois and H. Prade, Fuzzy Sets and System: Theory and Applications. Academic Press, New York, 1980.

[11] G.J. Klir, U.S. Clair and B. Yuan, Fuzzy Set Theory: Foundation and Application. Prentice Hall, Upper Saddle River, NJ, 1997. 